\title{
Giant Adrenal Lipoma with Hemorrhage Requiring Extended Surgical Resection
}

\author{
Kanji Otsubo ${ }^{a}$ Shinjiro Kobayashi ${ }^{a}$ Keisuke Ida ${ }^{a}$ Masafumi Katayama ${ }^{a}$ \\ Satoshi Koizumi ${ }^{a}$ Junki Koike ${ }^{b}$ Takehito Otsubo ${ }^{a}$ Atsuhito Tsuchihashi ${ }^{a}$ \\ aDepartment of Gastroenterological and General Surgery, St. Marianna University School

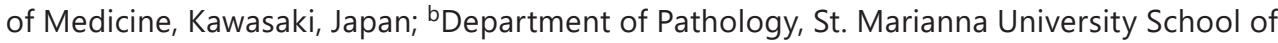 \\ Medicine, Kawasaki, Japan
}

\section{Keywords}

Adrenal lipoma $\cdot$ Hemorrhage $\cdot$ Distal pancreatectomy

\begin{abstract}
Adrenal lipoma is a rare, benign tumor, reported to account for $0.7 \%$ of primary adrenal tumors. A 69-year-old man presented with left lateral abdominal pain. Computed tomography (CT) was performed, and a huge, irregularly shaped retroperitoneal tumor of uneven internal density was identified, with the border between the tumor and the pancreas and kidney being unclear. Active hemorrhage was also depicted. The tumor consisted mainly of fat, with the exception of the hematoma; it measured $200 \mathrm{~mm}$; and the boundary between it and nearby organs, such as the pancreas, was unclear. Despite angiography being performed twice, the responsible vessel was not identified. Thus, for the purpose of both diagnosis and treatment, we resected the tumor, and considering the possibility of a malignancy, such as liposarcoma, we also resected the pancreatic body and tail and the spleen. The final histopathologic diagnosis was benign adrenal lipoma with hemorrhage, with no invasion to surrounding tissue. Hemorrhage within an adrenal tumor is rare. Most adrenal lipomas are small "incidentalomas" and asymptomatic. With development of a large adrenal lipoma comes the possibility of hemorrhage along with the possibility of features suggestive of malignancy. We encountered a giant adrenal lipoma with hemorrhage and, because of the aforementioned features, performed extended surgical resection, seen in retrospect as oversurgery. The widespread use of $\mathrm{CT}$ has led to an increased number of reported cases of adrenal lipoma. We anticipate an accumulation of case reports, which will allow for development of an appropriate treatment algorithm.
\end{abstract}




\section{Introduction}

Adrenal lipoma is an uncommon benign mesenchymal tumor that arises from the adrenal cortex. Most adrenal lipomas are asymptomatic and detected incidentally during imaging performed for another indication, but some present with abdominal pain, depending on their size [1-3]. Intratumoral hemorrhage has been reported in 2 cases of adrenal lipoma [2, 4], and retroperitoneal bleeding has been reported in 1 case [5], and all 3 were treated by simple adrenalectomy. We describe our experience in diagnosing and treating a giant adrenal lipoma with hemorrhage by means of extended resection. We highlight features of the case that made diagnosis and treatment decisions difficult.

\section{Case Report}

A 69-year-old man, having experienced sudden left abdominal pain, was referred to us after first visiting another hospital. His medical history included hypertension and a left lower leg fracture. Upon admission to the other hospital, physical examination revealed no abnormality other than left abdominal pain. Contrast-enhanced computed tomography (CT) revealed a huge, irregularly shaped retroperitoneal tumor of uneven internal density, with the border between the tumor and the pancreas and kidney being unclear (shown in Fig. 1). Active hemorrhage was also depicted. On the CT image, the tumor appeared large, measuring $200 \times 170 \times 100 \mathrm{~mm}$. Borders between the mass and surrounding tissues, such as the pancreatic body, pancreatic tail, and spleen, were unclear. Transarterial embolization was tried, but the culprit artery was not detected, so the patient was transferred to our hospital.
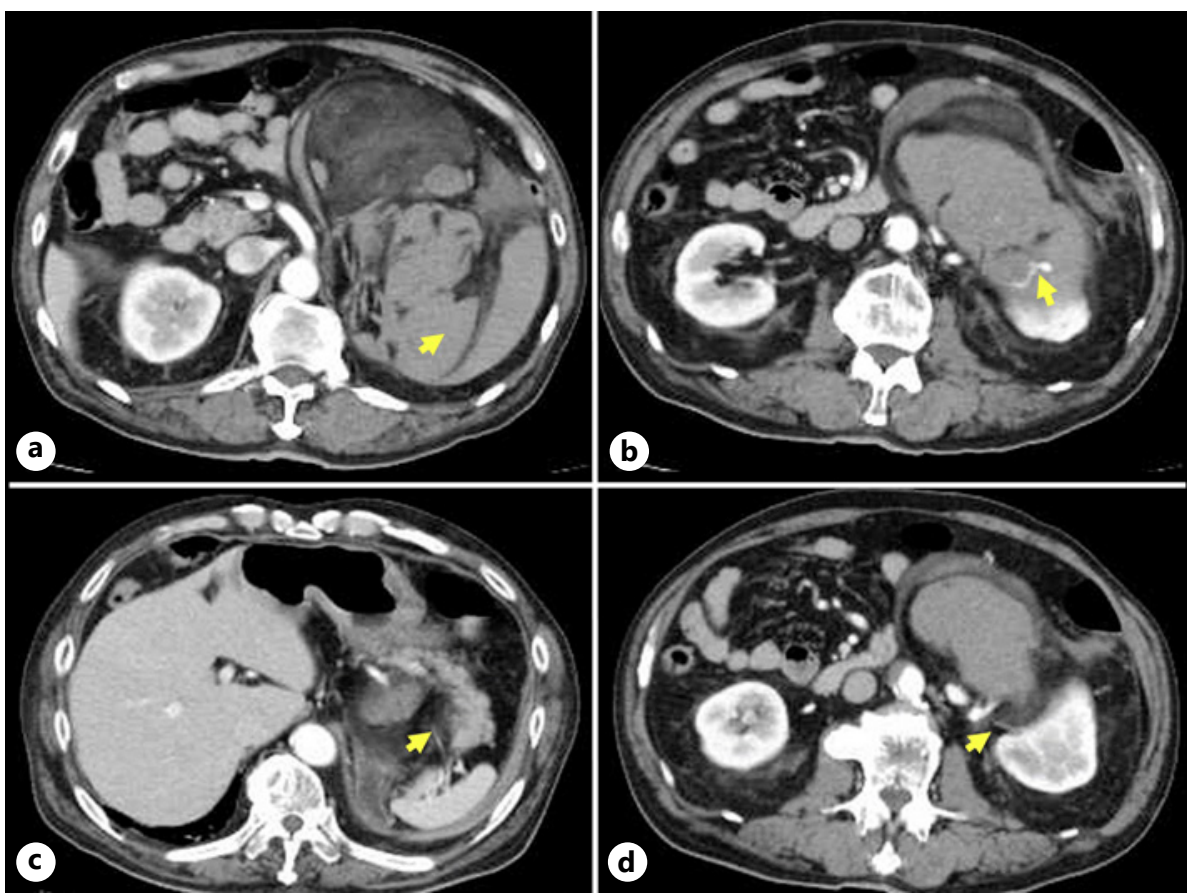

Fig. 1. Contrast-enhanced abdominal computed tomography performed on admission revealed a tumor in the retroperitoneum, $20 \times 17 \times 10 \mathrm{~cm}$ in size (shown by arrow in a). Active bleeding was seen within the tumor (shown by arrow in $\mathbf{b}$ ). The border between the tumor and pancreatic body and tail appeared fuzzy (shown by arrow in c) as did the border between the tumor and left kidney (shown by arrow in d). 


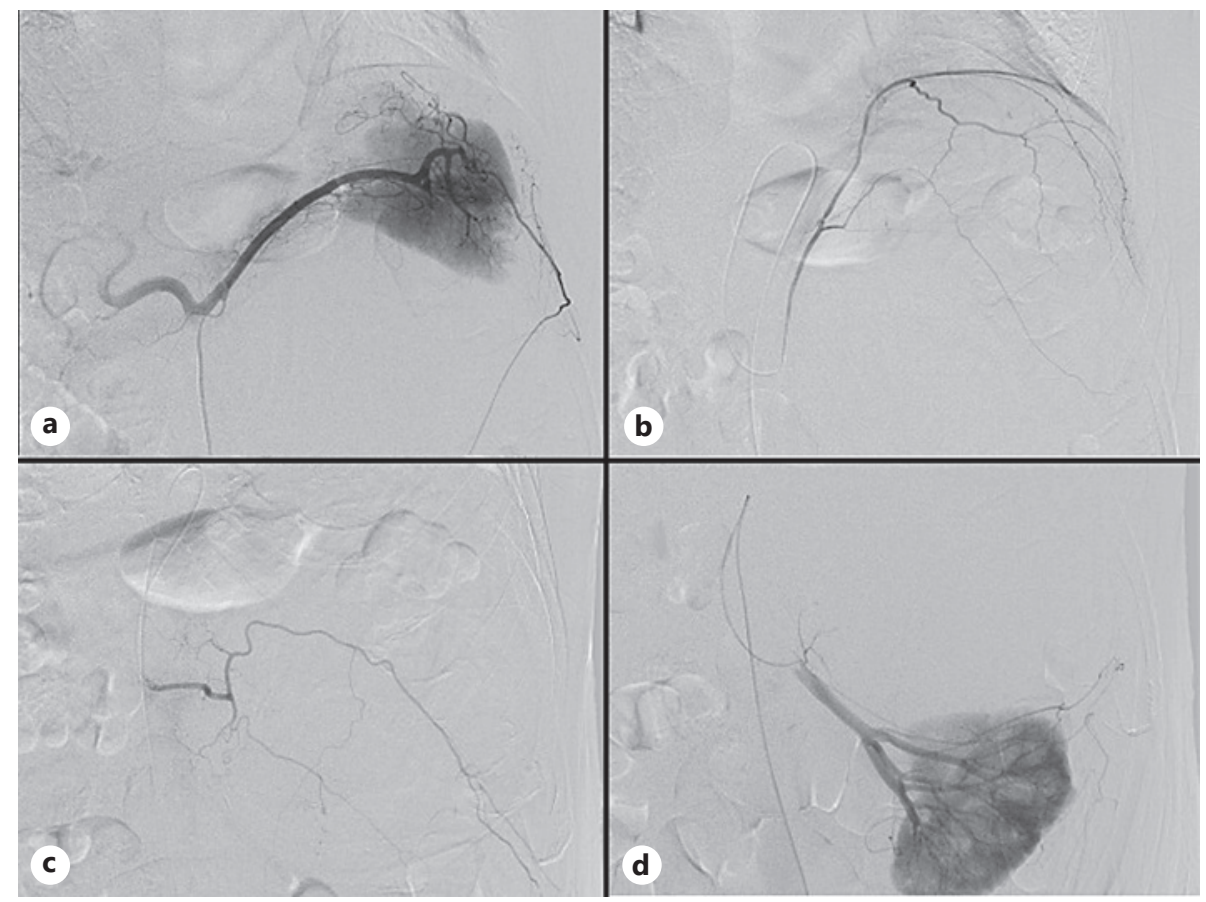

Fig. 2. Angiography showed no extravasation from vessels feeding the adrenal tumor, that is, the splenic artery (shown in a), left phrenic artery (shown in $\mathbf{b}$ ), left adrenal artery (shown in $\mathbf{c}$ ), and left renal artery (shown in d).

Our hospital's interventional radiology team attempted to localize the source of the bleeding angiographically but were not able to do so (shown in Fig. 2). CT showed no active bleeding, so we presumed the bleeding had stopped spontaneously by bed rest. For both diagnosis and treatment, we planned a semi-emergency operation. Considering the possibility of a malignancy, such as a liposarcoma, and of strong adherence of the tumor to the body and tail of the pancreas, we planned tumor resection combined with distal pancreatectomy. Intraoperatively, the giant tumor was located in the retroperitoneum cranial to the transverse colon and found to strongly adhere to the body and tail of the pancreas. In addition to resecting the left adrenal gland, we performed distal pancreatectomy, resecting the pancreatic body and tail and spleen, as planned.

On histopathologic examination, the tumor measured $180 \times 160 \times 90 \mathrm{~mm}$. Extensive hemorrhage and necrosis were seen, and liposarcoma and adrenal cancer were included in the differential diagnosis. Macroscopically, an obvious fibrous capsule enveloped the tumor, and no invasion of the pancreas or spleen was seen (shown in Fig. 3). Microscopically, the tumor was found to be composed of mature adipose tissue without nuclear atypia or mitoses and partially surrounded by a thin layer of adrenal cortex (shown in Fig. 4). Inflammatory cells, that is, giant cells, lymphocytes, and plasma cells, had invaded tissue surrounding the adrenal gland. Immunohistochemistry was negative for CDK4 and MDM2, which suggested the tumor was not a liposarcoma. The final diagnosis was adrenal lipoma with hemorrhage.

The postoperative course was good, and the patient was discharged on postoperative day 15. A little over 2 years has passed since the surgery, and there have been no signs of recurrence. 


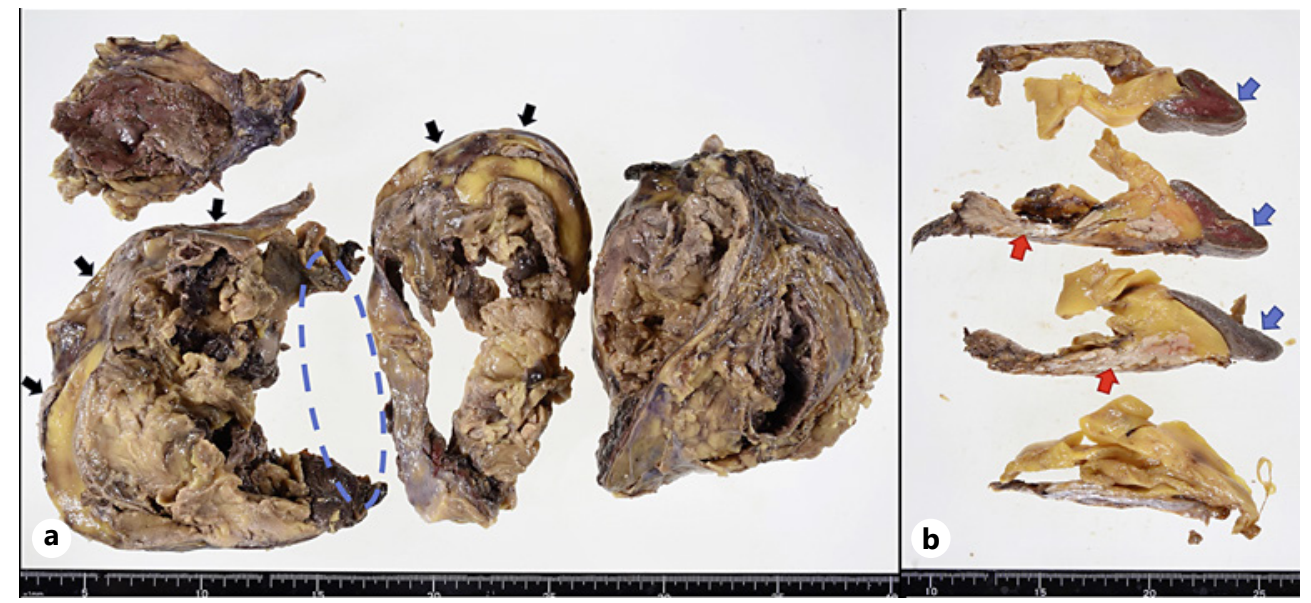

Fig. 3. Macroscopic examination of a transverse section of the resected tumor revealed a fibrous capsule (shown by black arrows in a) and partial collapse on the posterior surface having adhered to the left kidney (shown by blue circle in a). There was no invasion into the pancreas (shown by red arrows in $\mathbf{b}$ ) and spleen (shown by blue arrows in b).

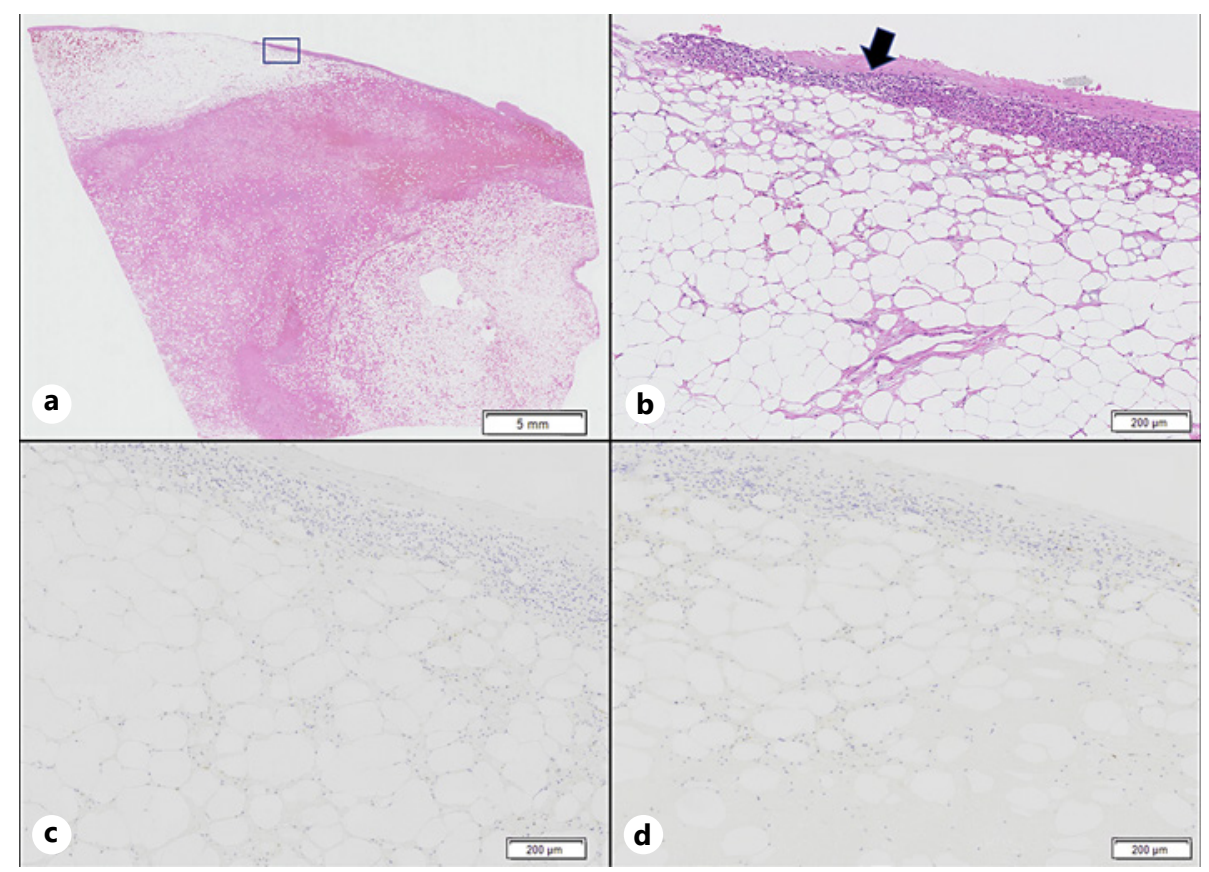

Fig. 4. Histopathologic examination revealed severe hemorrhage and fat necrosis. The tumor consisted, for the most part, of mature adipose tissue (shown in a). A thin layer of the adrenal cortex was evident just below the capsule (shown by arrow in b). There were no lipoblasts suggestive of sarcoma. There was no atypia, and there were no myeloid cells. Immunohistochemistry was negative for CDK4 (c) and MDM2 (d).

\section{Discussion}

Adrenal lipoma is a rare adrenal tumor that accounts for $0.7 \%$ of primary adrenal tumors and $4 \%$ of adrenal fatty tumors [6]. According to the 2017 WHO update [7], adrenal lipoma is more common in males than in females (male-to-female ratio $=1.0$ to 0.7 ), it tends to occur 
in the sixth decade of life (mean age $=54$ years [range $=35-78$ years]), and it also tends to occur on the right side (right-to-left ratio $=2.4$ to 1 ). The average major axis is $75 \mathrm{~mm}$, with the axis ranging from 10 to $200 \mathrm{~mm}$. Two-thirds of patients are Asian. As noted above, 3 cases of bleeding from an adrenal lipoma have been reported, and all of them were treated by an open or laparoscopic procedure (Table 1). There are some reports of bleeding from lipomas in the digestive tract $[8,9]$. Large adrenal lipomas are prone to mechanical stimulation due to peristalsis, and this stimulation can lead to ulcers and bleeding. Thus, surgery is often indicated for a large lipoma.

Here, we report a case of intratumoral hemorrhage in a case of left adrenal lipoma that developed in a 69-year-old Japanese man. Two points stand out: although adrenal lipomas are often found by chance, they sometimes present with pain due to bleeding. Also, because malignancy cannot always be ruled out on the basis of imaging findings, such as bleeding and an unclear border between the tumor and surrounding structures, there may be a need to plan extended resection.

As noted, adrenal lipomas rarely cause bleeding. According to recent reviews, most adrenal lipomas are asymptomatic [7]. All 4 cases of hemorrhage, including ours shown in Table 1, presented local pain, and the tumors averaged $140.5 \mathrm{~mm}$ in size and were larger than the overall average of $75 \mathrm{~mm}$. It can be said that large adrenal lipomas are prone to bleeding and local pain. Considering the tendency of large adrenal lipomas to bleed, it is thought that when such a tumor becomes large, blood flow develops, and intratumoral bleeding is likely to occur as the result of some kind of stimulus.

There are 2 differences between our case and the 3 previously reported cases of adrenal lipoma with hemorrhage in Table 1. First, in our case, the tumor was $200 \mathrm{~mm}$ in size, which was even larger than the 3 previously reported cases. Second, the 3 of them had smooth margins on CT and no adhesions to surrounding tissues in macroscopic findings, whereas in our case, the boundaries between the tumor and surrounding tissues were unclear on CT and admitted strong adhesion to surroundings. The mechanism of this difference can be described as follows. With the relatively rapid and heterogeneous growth of the tumor, there arises some areas which has insufficient blood flow due to stress of extension and compression, resulting in necrosis followed by intratumoral hemorrhage. With further tumor growth, rupture of the tumor wall causes retroperitoneal bleeding. The bleeding component contains many inflammatory cells that have infiltrated due to the phagocytosis of necrotic tissue, resulting in strong inflammation and adhesions around the tumor. Therefore, it can be said that the larger the tumor, the worse the bleeding and adhesions.

An adrenal lipoma with hemorrhage possibly adheres to surrounding tissues and not be easily distinguished from a malignancy, such as liposarcoma. In such cases, extended surgery may be indicated. In our case, although CT showed the tumor to consist almost entirely of fat, a malignant component could not be ruled out because the tumor was huge and intratumoral hemorrhage was present. Liposarcoma recurs at a high rate [10]. To achieve complete resection, we removed not only the adrenal gland but also the surrounding tissue and adjacent organs but without performing adhesiolysis. Therefore, we resected the pancreatic body and tail and spleen along with the adrenal lipoma. The final pathologic diagnosis was adrenal lipoma, which is a benign tumor, so one can say avoidance of oversurgery is difficult in such cases.

Adrenal lipoma is a rare, benign tumor. If a large adrenal lipoma develops, hemorrhage can occur. The border between a giant adrenal lipoma with hemorrhage and surrounding organs may be unclear, so there are cases in which extended surgery should be performed, due to the possibility of liposarcoma. The widespread use of CT has led to an increased number of incidental detection of adrenal lipoma. If the tumor can be detected earlier, intratumoral hemorrhage and adhesions due to retroperitoneal bleeding will be reduced, and simple adrenal resection could be possible.

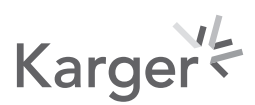


Case Rep Gastroenterol 2021;15:978-984 \begin{tabular}{l|l}
\hline DOI: 10.1159/000520626 & @ 2021 The Author(s). Published by S. Karger AG, Basel
\end{tabular} www.karger.com/crg

Otsubo et al.: A Case of Giant Adrenal Lipoma with Hemorrhage

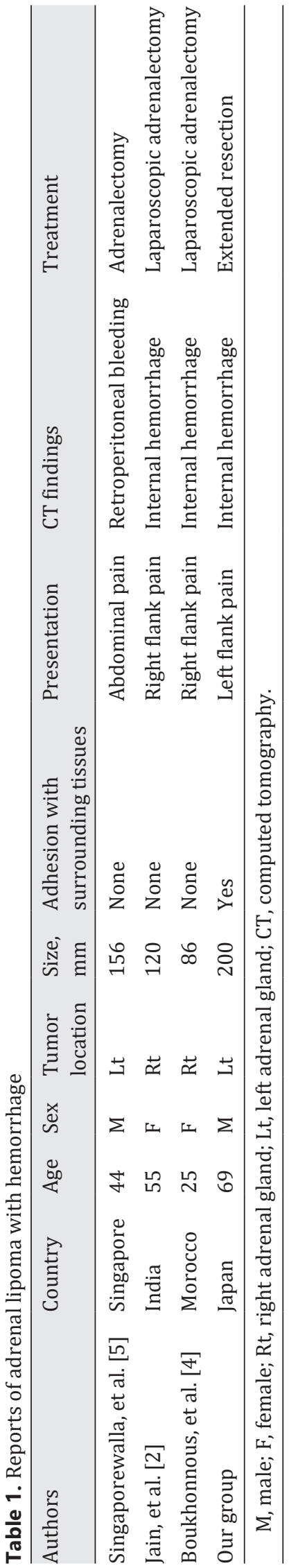

Karger' 


\section{Statement of Ethics}

All the procedures followed have been performed in accordance with the ethical standards laid down in the 1964 Declaration of Helsinki and its later amendments. This study protocol was reviewed and the need for approval was waived by the Bioethics Committee of St. Marianna University School of Medicine. Written informed consent was obtained from the patient for publication of this case report and accompanying images.

\section{Conflict of Interest Statement}

The authors have no competing interests directly related to the content of this article.

\section{Funding Sources}

This manuscript did not receive any funding.

\section{Author Contributions}

S.K. (Shinjiro), A.T., R.K., M.K., S.K. (Satoshi), and T.O. were involved in the patient's treatment. J.K. performed the histopathological examination. K.I. was instrumental in the composition of the text and the interpretation of the figure. All authors read and approved the final manuscript.

\section{Data Availability Statement}

All data generated or analyzed during this study are included in this published article. Further enquiries can be directed to the corresponding author.

\section{References}

1 Sharma MC, Gill SS, Kashyap S, Nabi G, Mishra MC. Adrenal lipoma. A case report. Urol Int. 1998;60:245-7.

2 Jain D, Chopra P, Sharma A. Adrenal lipoma with hemorrhage: a cause of abdominal pain. Urol J. 2012; 9:721-4.

3 Abe T, Kawamura S, Aoki H, Kubo T, Monoma N. [A case of adrenal lipoma]. Nihon Hinyokika Gakkai Zasshi. 1994;85:1563-6.

4 Boukhannous I, Aynaou M, Mhanna T, Houmaidi AE, Miri A, Barki A. Hemorrhagic adrenal gland lipoma: about a rare case and review of literature. Urol Case Rep. 2020;33:101294.

5 Singaporewalla RM, Thamboo TP, Rauff A, Cheah WK, Mukherjee JJ. Acute abdominal pain secondary to retroperitoneal bleeding from a giant adrenal lipoma with review of literature. Asian J Surg. 2009;32:172-6.

6 Lam KY, Lo CY. Adrenal lipomatous tumours: a 30 year clinicopathological experience at a single institution. J Clin Pathol. 2001;54:707-12.

7 Lam AK. Lipomatous tumours in adrenal gland: WHO updates and clinical implications. Endocr Relat Cancer. 2017;24:R65-79.

8 Yatagai N, Ueyama H, Shibuya T, Haga K, Takahashi M, Nomura O, et al. Obscure gastrointestinal bleeding caused by small intestinal lipoma: a case report. J Med Case Rep. 2016;10:226.

9 Kim JH, Yoon HH, Jeong SH, Woo HS, Lee WS, Choi SJ, et al. Spontaneous peeled ileal giant lipoma caused by lower gastrointestinal bleeding: a case report. Medicine. 2017;96:e9253.

10 Singer S, Antonescu CR, Riedel E, Brennan MF. Histologic subtype and margin of resection predict pattern of recurrence and survival for retroperitoneal liposarcoma. Ann Surg. 2003;238:358-1; discussion 370-1.

\section{Karger'}

